\title{
Health as a genetic planning project: Enthusiasm and second thoughts among biomedical researchers and their research subjects
}

\author{
STEFÁN HJÖRLEIFSSON, ROGER STRAND \& EDVIN SCHEI
}

\begin{abstract}
This paper presents an interview study among scientists working with Decode genetics in Iceland and lay individuals having recently donated blood to Decode. While genuinely enthusiastic that genetic technologies hold great potential to avert disease, the informants shared concerns that extensive predictive genetic testing, preventive treatment and tailoring of lifestyle to avoid potential disease may cause loss of freedom - people can "worry themselves sick". Undiscriminating use of genetic technologies in privileged populations was seen as a potential source of injustice and reduced tolerance of diversity. Both lay informants and scientists revealed ambiguity and inconsistency in their personal evaluation of genetic knowledge, indicating that 'rational choice' models do not predict how people relate to information about risk, expert knowledge notwithstanding. Drawing on work by Wynne and van Hooft, we submit that our informants' ambivalence and second thoughts are implicit contradictions of prescriptive messages accompanying human genetics - i.e. more or less covert and non-intentional claims about the rational obligation to minimise the likelihood that one falls ill and a strictly biological conception of health. Genetic technologies designed to prevent or combat organic disease can interfere negatively with non-biological levels of health. It is a challenge of reflexive modernity to untangle the interaction of human genetics with culturally mediated categories of relatedness, purpose and meaning in everyday life, and mobilise cultural and governance resources which can ensure that genetic technologies support human subjectivity and health in their full range.
\end{abstract}

\section{Introduction}

Public debate on human genetics can be both polarized and polarizing. On one hand, a significant subset of the public in many countries is wary of many uses of human genetics and certain other biotechnologies. On the other, dominant science policy discourse - apparently supported by the majority of the public - expresses a general approval of these technologies as a major source of improved health, quality of life and economic growth, with a few exceptions such as human reproductive cloning. ${ }^{1}$ Mass media appear to reinforce polarization by their very repertoire in the framing of human genetics. There is the frame of technological optimism, emphasising scientific bravery and reproducing scientific promises of relief from disease and suffering. ${ }^{2}$ Alternatively, if the technology in question is somehow controversial (such as reproductive cloning), a frame of fear and horror is easily applied, evoking the Frankenstein myth, as it were. ${ }^{3}$ A similar polarization tends to be conjured by the expert and media portrayal of public sentiments, as in the case of GM food, held by British media and expert reports to divide the public into mutually exclusive fractions. $^{4}$ 
Nevertheless, contemporary societies arguably have made the transition into reflexive modernity, in which citizens recognize and debate the joint production and redistribution of benefits and risks as an inherent aspect of scientific and technological advance. Hence, people's sentiments towards genetic technologies are likely to be more nuanced, reflexive and ambivalent than what is conveyed by policy debate, mass media or even by surveys. ${ }^{5}$ The marginalization or privatization of reflexivity may in part be due to the dominance of technological optimism in the policy discourse about healthcare and genetic research. Despite evidence about the shortcomings of the socalled 'deficit model', second thoughts and reservation regarding genetic technologies are frequently de-legitimised in public debate by blaming them on ignorance. But marginalization may also be an artefact of reporting, as mainstream media rendering of quantitative surveys does not easily capture complex reasoning, nuanced sentiments or ambivalence.

This paper presents an exploration of reflexivity through a qualitative study among individuals knowledgeable and prima facie supportive of human genetics in Iceland. We believe this task to be important. Discrepancies between lay and expert perception of human genetic technology, and more subtly, between real and presumed perceptions, may easily translate into an erosion of public legitimacy similar to that which has shaken the governance of related technologies in recent years. ${ }^{6}$ Further, unacknowledged reflexivity will not feed into processes of governance, increasing the danger that technology will cause more harm than benefit. This raises the need to understand the public understanding of genetics as well as the democratic challenges of developing inclusive governance taking that understanding into account.

In Iceland, the private enterprise Decode genetics has collected data from more than $50 \%$ of the adult population for their research into the genetic components of many common diseases, including myocardial infarction, schizophrenia and asthma. Following a fierce debate locally and internationally in 1997-2000 about Decode's plans for a nation-wide database with comprehensive healthcare information, the company recently appears to have achieved a favourable position in the Icelandic social and cultural imagery. ${ }^{7}$ According to a Eurobarometer conducted in 2005, the Icelandic public is more supportive of biobank research and biotechnology than people in any other European country, ${ }^{8}$ and according to the company itself more than $90 \%$ of the public respond favourably when asked to contribute to Decode's research projects. ${ }^{9}$ Our hypothesis was that eliciting reflections in this setting, among individuals knowledgeable and supportive of human genetic research, would allow a philosophical-anthropological analysis of different and potentially conflicting meanings attached to genetic technologies, in particular with respect to personal health risk management. Accordingly, we performed focus group interviews about the impact of human genetics on everyday life with scientists working with Decode genetics, as well as with individuals who recently had contributed to Decode's research, i.e. donors of blood samples and personal information.

This study forms part of a larger investigation into the reactions to Decode genetics and the governance of human genetics in Iceland. ${ }^{10}$ It is our opinion that the challenge of reflexive modernity is to pass into pre hoc anticipation of higher-level effects of genetics to improve the governance of novel technologies. By 'higher level effects' we mean the socio-cultural repercussions of genetic research and the associated 
technologies. Before presenting and discussing the results of our focus group interviews, we will outline a general framework for the higher-level effects of science and technology as found in the work of Brian Wynne, followed by a brief account of Stan van Hooft's theory of health, which we take to provide a useful conceptualisation of some of the most central dimensions of culture interrelated with the current rise of human genetic research.

\section{Theoretical framework}

Wynne writes that public reactions to science and technology concern not only the concrete facts, proposals and applications, but also the 'prescriptive messages' of technological projects. ${ }^{11}$ These are value-laden messages about human agency, the nature of human existence, the role of science in the broad context of everyday life, etc., that accompany science and technology as their presuppositions or implications. The transmission of such messages is a hermeneutic process, resulting not only from more or less deliberate intentions originating in expert institutions, but also through a dialectic of lay interpretations, reflecting a variety of knowledge, values, fears and hopes. According to Wynne, public interpretation of the prescriptive models of human agency and risk management is germane to the public critique of science, as in the case of, say, hostility towards GM food and crops and technological solutions to environmental pollution. ${ }^{12}$

Following Wynne's line of reasoning, the subtleties of public perceptions of human genetic technologies are directly relevant to the implications of these technologies on health and health care. Already from Thomas' and Thomas' classic theorem, "If men define situations as real, they are real in their consequences", ${ }^{13}$ it follows that public perception will feed back into real action in society. In particular, we expect the practical lifeworld consequences of these technologies to be broader than the facevalue clinical utility, and to depend upon the ways in which they are understood and integrated into thoughts, expectations and actions. This integration is crucially linked with what Wynne calls prescriptive messages, and will depend upon a large number of factors including scientists', stakeholders' and media's appearance in terms of trustworthiness, competency and responsibility. However, integration also has to be seen as an essentially open-ended and unpredictable process in which the public and society perform a creative translatory work upon the technologies, their prescriptive messages, and their own world-views and value systems.

A central lifeworld dimension involved in the rise of human genetics is health itself. By promising and providing new knowledge about disease mechanisms, risk, and the potential consequences of a person's attitudes towards genetic tests, lifestyle and reproduction, genetics unleashes an unlimited number of intended and unintended 'messages' which interact with people's thoughts, actions, relationships and identities. Conversely, contemporary beliefs about human agency, disease and the power of medical interventions form the cultural environment which enables and gives shape to the rapid development of genetics. To deal with certain features of this interaction, we shall apply the broad concept of health developed by van Hooft, in which health is seen as inseparable from human subjectivity and as such inherently value-laden and conditioned by the particularity of individual persons, embedded in the language and culture of late modernity. ${ }^{14}$ The crucial feature of van Hooft's account in our context 
is that it expands the biomedical model of health by identifying four levels of subjectivity, referring to different aspects of human existence, from molecule to the meaning of life, where suffering and loss of health may arise. The first level is the biological and concerns the functioning of organs, tissues and molecules. The second, relational, level denotes the ways in which we relate to reality through perception and interpretation, depending on largely unconscious processes of meaning-construction. The third, pragmatic, level concerns people's ability to fulfill social roles and plan their lives. The fourth level, integration, denotes our need for self-understanding, purpose and belonging, our dependence on community and narratives that help us commit ourselves and see life as worthwhile. Although the three non-biological levels of health can be distinguished from each other for analytical purposes, in reality they tend to be integrated with each other and with the biological dimension. For our present purpose, distinguishing the non-biological dimensions of health from biological health will be crucial. It is easy to see that activities focusing solely on one level may result in unanticipated effects on a different level. Indeed, the history of medicine bears evidence of numerous instances where medical interventions effectively targeting the biological level also had unpredicted health effects on the three other levels. Certain quarantine and eugenic practices are but two examples of practices that were abolished not primarily because they were ineffective or harmful to health on the biological level, but because they were found to have detrimental effects on the three other levels. Also, a significant reason for the current rise of alternative therapies in societies with advanced biomedical healthcare services seems to be the tendency of biomedical practices to focus on the biological, while not adequately addressing higher levels of subjectivity. With regard to human genetics, a challenge of reflexive modernity is to develop the means to conceptualise and evaluate the higher-level effects of these technologies.

\section{Method and material}

As the aim of the study was to search for ambivalence and reflexivity among individuals who in one way or another were committed to the utility of human genetics, we chose to interview these individuals in focus groups of 4-5 participants each. While the group setting provides a dynamics and a certain anonymity as compared with individual interviews, these small groups were more suitable for establishing the trust needed to discuss ambivalence than the conventional focus groups of 6-12 participants would have been. 13 scientists working with Decode were purposefully selected to achieve a fertile exchange of opinions and beliefs. To complement this material, we recruited four consecutive individuals among those volunteering blood samples and healthcare information to Decode. ${ }^{15}$

Focus group interviews lasting two hours were carried out by $\mathrm{SH}$ in four groups; two groups of 4 and one consisting of 5 scientists, the last one consisting of the 4 lay participants. A semi-structured approach was used, based on a short interview guide and basic principles for focus group interviews. The informants were asked to discuss any likely consequence of human genetic research in the lives of 'ordinary people'. They were encouraged to use examples from their own experience, and it was explained that the aim of the discussion was to explore rather than necessarily to agree on any definite views of the topic given. The interview guide included a strong commitment to take seriously any suggestions and points of view submitted by the 
informants, i.e. encouraging an earnest discussion of their merits, the relationship between different suggestions, etc.

The interviews with the scientists were conducted in English, while the lay participant interview was conducted in Icelandic. Full audiotape recordings were transcribed verbatim, with the recording from the lay participant interview being translated into English at the time of transcription. All transcripts were coded for emerging themes by SH and then analysed. The transcripts were subsequently coded and analysed by RS, and finally all three investigators agreed on a common interpretation after trying diverging interpretations against each other as well as against the theoretical framework, also seeking to take account of how the investigators' preconceptions might influence the analysis. ${ }^{16}$

\section{Results}

The results are presented under three headings, each section being introduced by typical quotes from the interviews.

\section{Benefits, with epistemological, clinical and practical limitations}

There is so much hope that this will be the key to everything. But I think we will never get there. You know, we are hoping that this could be a good diagnostic tool, that we will be able to develop drugs for everything that we will be able to diagnose. And then to correct diseases with gene therapy... If we're talking say 15-20 years we will know maybe a little bit more about things and ... but I think to be able to cure things ... it's far, far away from us. Even though we have all those tools it's going to be ... But it can be helpful in some cases to be able to, like with pharmacogenomics to be able to tailor the drug therapy. ... I think the steps are all going to be very small. For example with the human genome project, when, you know, all the human DNA had been sequenced, people really hoped that it would, you know, open the door to almost everything. But it is a very small, minor step ... that we just know the letters in there, but we don't know what they mean (scientist).

Our informants - lay and scientists - all expressed the view that human genetics will bring great benefits to healthcare. The scientists were enthusiastic that their research was showing promising results, and they were confident that research will translate into healthcare benefits. The lay participants stated without hesitation that the research they were contributing to was important. This was the unmistakable 'baseline' point of view among our informants, and a major finding against which our subsequent results have to be evaluated. The reservations, concerns, second thoughts and ambivalence that we present below gain their relevance against this background.

Scientists and lay participants alike were unanimous that progress would be slow, as the way from basic genetic research to substantial clinical benefit is long and tortuous. The informants identified and discussed a catalogue of epistemological, clinical and practical limitations of genetic prediction and preventive measures derived from genetic information. Here, we will focus mainly on predictive testing. 
First, the epistemological value of predictive information derived from genetic testing will be limited, especially when it comes to testing for complex diseases and traits: Several scientists stated that it is "quite clear that predictions are uncertain" in the sense that even if an individual is found to carry a genetic disposition for a given disease it is far from certain that he or she is going to develop that disease. As an example of this, if Decode or others were to identify a genetic polymorphism correlated with alcohol abuse, there would be "a lot of people with this alcoholismgene who are not alcoholics", according to one of the lay participants.

Secondly, it follows from the epistemological characteristics of predictive information that using this information as the basis of clinically relevant advice about lifestyle and preventive treatment is not straightforward. It is clearly a problem that diagnostic and prognostic technologies tend to be introduced ahead of evidence of their accuracy or the effectiveness of intervention. In addition it is unlikely that any preventive treatment will completely eliminate a given genetic risk. Also, a negative test result does not mean zero risk, and therefore leading a healthy life is important in any case. There will be uncertainty about potential genetic risks that have not been mapped, as stated by a scientist talking about a disease which runs in his family: "I would just be thinking 'is there another gene?"' The scientists agreed that making sense of genetic information and turning it into wise choices in everyday life would remain a hard task for most people, especially as information becomes more complex. When provided with information about multiple risk factors, people will have a hard time figuring out and adapting to the information: "[O]ne can wonder to what extent people will be able to understand these tests and to make use of them". The scientists also expected that the importance of genetic predispositions would be overestimated in some instances: "Some people might just go to bed and think, well my life is over, I have a three times risk of getting ..." Some of the scientists wondered optimistically whether information technology could be used to 'translate' genetic information so that people could form appropriate opinions despite lacking the background knowledge necessary to evaluate the technical details of risk estimation and risk reduction. Then again, not everyone has the motivation to change lifestyle in accordance with medical evidence. Actually, the general knowledge that people should exercise, and avoid smoking and excessive consumption of food is available to everyone already, and still the lifestyle of the general public in most societies is becoming unhealthier by the minute. Some of the scientists wondered that this unfortunate trend might escalate as it becomes coupled with widespread use of lifestyle drugs designed to minimise the risk imposed by self-indulgence and inheritance. Unfortunately, the scientists said, many "people would rather "pop a pill' than go to the gym". The misgivings of the scientists were largely corroborated by the lay participants. The avalanche of information from genetic research will be "an extremely large package" to people, as one of them put it.

\section{Impact on dignity, tolerance and justice}

When these individuals disappear from society, it will be: I'm precious ... my own needs. It will fail ... the sense of humanity. All this raises the need to sort out in general what is quality of life (lay participant). 
The lay participants expressed a concern that genetic testing in pregnancy will increase the pressure to 'eliminate' abnormal foetuses. Although Decode has emphasised technologies targeting postnatal prediction and prevention of disease, the lay participants found it natural to include a discussion about reproductive technologies and suggested that dignity and humaneness are linked with diversity and tolerance in ways which make it dangerous for society to systematically 'eliminate' deviant individuals such as dwarfs and people with Downs syndrome.

The lay participants also wondered whether genetic research, although potentially useful, should be prioritised above other measures. When preventive measures based on genetic technologies are introduced, some people are going to demand them regardless of whether they are cost-effective in comparison with other interventions. The scientists discussed along the same lines that new technologies are demanding on resources, and genetic technologies may add to inequalities in health across social and global gradients. The possible creation of a 'genetic elite' was discussed, including references to popular culture (as in the film 'Gattaca') and popular science (as in the book 'Remaking Eden' ${ }^{17}$ ). All of this was seen as unjust and unfortunate.

\section{Fighting disease and taking care of one's own health}

I'm thinking about my [children]. How can I tune [their] life to avoid the stuff that are true risk factors today? Of course I want to understand this better. Give them proper food when they grow up, so they won't have the deficiency or whatever ... signalling substance (scientist).

And then it's the question ... er ... do you feel any better if you have this list from the moment you're born with all the syndromes that [starts to laugh quietly, the rest of the group gradually join] ... or, I don't know ... or that you might get? You're at high risk for this, and this and that. And if you are either going to live by this or oppose it and ignore it completely, what the heck, I'll just get everything. I don't know if that's a kind of knowledge that would actually be useful for one (lay participant).

I think you have to balance the risk factors with what you can call the quality of life. You can worry yourself sick if you know too much (scientist).

\section{A. Medical rationality and the empowering function of knowledge}

The scientists sometimes argued as if medical rationality were the ultimate guide to a successful life. One should keep informed about one's dispositions to disease and make informed choices about lifestyle and preventive interventions. A scientist who until recently had been working as a MD, assured that this was how he used predictive information in his work, giving his patients "very concrete instructions about how they should behave and not behave in terms of various risk factors." The scientists said they hoped their research would lead to the discovery of genetic dispositions to disease that would allow people to take appropriate measures to avoid disease: "If we could identify those major players, then people could tailor their life." They emphasised the empowering capacity of predictive knowledge, i.e. that people can be put "in a more empowered role of being able to do something about it should they choose so." 
The general rule that one should follow medical advice was made more tangible when the scientists described how they would like to 'tailor' their own life or that of their children through use of medications, lifestyle-interventions and specialised diets, once "the true risk factors" become known. In one of the groups two female scientists also stated that if they were to learn that they were genetically predisposed to osteoporosis, they would respond by having their bone mineral density measured more frequently.

\section{B. Personal inconsistencies}

In discussions about how the scientists themselves were currently dealing with issues of risk, it became evident that they are not always compliant with medical rationality as described above. For example, two of the scientists submitted that they were not having their cholesterol measured, even if they were positive that this would be prudent. Similarly, it was not always clear to the scientists that they would choose to avail themselves of preventive medications, though they expressed enthusiasm and support for research programmes designed to develop such therapies. For example, one of the scientists stated that she probably would not wish to take medications to prevent stroke, in spite of the fact that several of her older relatives have suffered from that disease: "I'm dying to know if I have that haplotype or not, but what I would do with it? I would not really change my life or anything. But would I take a pill to prevent ... based on that haplotype? No." In much the same way, the lay participant group was not willing to conclude that the teenage daughter of one of the participants would be better off knowing about whatever genetic dispositions she might be carrying.

The discursive devices employed by the scientists to explain apparent inconsistencies between the ideal of medical rationality and their own choices, included judgements about "stupidity and laziness", failure to make one's priorities correctly or inconvenience. These explanations were frequently coloured by laughter, signalling as well as containing unease about one's own shortcomings.

\section{C. The limits of medical rationality}

On several occasions scientists and lay participants alike described potentially harmful effects of predictive knowledge and extensive preventive measures, and stated that prediction and prevention must not be made the only measure of how people lead their lives. This point of view is summarised in the following quote from one of the lay participants: "I think that people are going to ignore it, or I hope so [starts to laugh, quietly, the others gradually join the laughter]. That people don't live totally by ... Because you have got to have a little freedom." The scientists were clear that no matter how sophisticated technologies that are developed, we will "never be free of the worries of being human with all its complications". Striving to eliminate every single worry will bring more misery than benefit. It did not become clear, however, under what circumstances this misery would outweigh the benefits of genetic technologies; and suggestions were made that people will gradually 'adapt' and learn how to make use of new information. In the following quote this is being discussed by a lay participant, but even here the use of laughter and the choice of Alzheimer's disease as an example seems to signal the unresolved nature of 'adapting' to predictive genetic knowledge: 
I think that one would just accommodate, just as everyone accommodates to knowing ... well we know that eventually everyone is going to get some disease. It always takes time to adapt to what is new, but I think that one would just be glad to know that yes, I'm at risk for this. Then you'd be waiting for the first symptoms [starts to laugh] yes [laughs louder, the others join]. Then you'd know what it was: Now the Alzheimer is coming!

\section{Discussion}

Although our informants demonstrated an ability to identify and discuss complex issues and concerns regarding the future of genetics, they may hold other beliefs on these topics than we have been able to elicit from these particular dialogues. Most obviously, the scientists may have had a strategic motive - perhaps encouraged by the interests of their employer - in portraying themselves as socially and morally responsible. Making a likeable and sympathetic impression is important to most people, and the scientists as well as the lay participants may have estimated that we the investigators - as well as the future readership of this report would approve if they gave the impression that they were concerned about potential harm caused by genetics. As stated above, however, we have approached our informants with a strong commitment to take seriously their arguments and stances, and in the following we will pursue the lines of thought communicated in the interviews without further speculation about vested interests.

Taking Wynne's idea about the prescriptive content of science and technology as our point of departure, the question to be answered is what we have learnt about how our informants relate to the prescriptive messages of human genetics. As indicated above, the organising principle of our results is that this relationship is characterised by an ambivalent mixture of enthusiasm, second thoughts and reservations. We will now discuss certain features of this mixture.

Our informants' general enthusiasm about the benefits of human genetics and the scientists' ideas about personal lifestyle choices and preventive treatment presented in section 3A illustrate a particular prescriptive model of human agency and the measures that should be taken to avoid disease. According to this model individuals are endowed with the capacity to make rational decisions based on objective knowledge about what is to their advantage or disadvantage. Based on predictive knowledge about their predispositions to disease, individuals are obliged by reason to make such decisions as minimise the likelihood that they fall ill. Each person's life should be 'tailored' with the aim of securing his or her health, and this is done by making the right lifestyle choices and benefiting from preventive medications in order to minimise specific disease risks. This prescriptive model, which we will label 'rational lifestyle choice' has strong foothold in research and healthcare policy in Western societies, and is part of the cultural milieu hosting and encouraging the rise of human genetics.

Already the acknowledgement of the limitations of predictive knowledge presented in section 1 of the results betrays that our informants are aware that this prescriptive model has serious limitations. Presupposing that people will make rational lifestyle choices based on information about a multitude of risks, that they enjoy the capacities 
needed to make all the 'right' choices, etc., runs counter to the common knowledge that awareness of lifestyle influences on health does not in itself guarantee that people 'tailor' their lives towards 'minimal risk'. In general, the very belief that there is a 'right' way of using genetic information, and the assumption that people can and will use genetic information in the 'right' way, are problematic. This emerges from the probabilistic nature of genetic information ${ }^{18}$ and the effects genetic technologies may have on the non-biological dimensions of health discussed by van Hooft. While genetic testing and preventive regimes increasingly provide opportunities for countering biological disease, the crucial insight is that these regimes also have the potential to interfere with the autonomous self-expression and spontaneity of individuals and societies which are essential ingredients of health in the nonbiological sense described by van Hooft. Deciding which are the 'right' conclusions concerning the use of preventive regimes based on genetic technologies is therefore likely to be a much more complex task than suggested by the prescriptive model of rational lifestyle choice.

The results presented in section 2 demonstrate our informants' attempts to explore how the prescriptive model implied in their most enthusiastic interpretation of genetics may impact other values and prescriptive beliefs, in particular the social virtues of dignity, tolerance and justice. Even if human genetics will increasingly improve biological health for privileged individuals, there is a danger of reduced tolerance of diversity and more generally a threat to the sense of meaning in people's lives, placing human dignity and sociality at peril. Under certain conditions the poor and underprivileged are likely to suffer increased injustice and deprivation in comparison with people enjoying good access to new genetic technologies. Although our informants did not dismiss genetic research on these grounds, or even conclude that human genetics is bound to cause intolerance and injustice, their discussion nevertheless illustrates that these adverse effects may occur, particularly if enthusiasm for human genetics is unrestricted and based on a particular set of prescriptive beliefs, valuing individual 'tailoring', risk reduction and enhancement to conform with narrowly conceived concepts of health and success. Thus, our results indicate that both lay people and scientists strongly in favour of genetic research and technology development, see the prescriptions of a narrow medical rationality as inadequate if genetic technologies are to be compatible with human dignity, tolerance and justice.

In sections $\mathbf{3 B}$ and $\mathbf{3 C}$ second thoughts and ambivalence are brought closer to our informants and their personal points of view. Some of the scientists betrayed that they were not personally following the lifestyle obligations to which they were otherwise eager to claim support. And neither the scientists nor the lay participants were certain that they would change their lifestyle or take medications in accordance with predictive knowledge based on genetic tests in the future. Within the rational choice model this amounts to nothing less than foolishness, and it is therefore understandable that our informants reacted by making fun of themselves and each others on these occasions. Especially for the scientists, any apparent failure to adhere to 'scientific standards' can easily become a cause of embarrassment. Acknowledging and underscoring one's humanness by use of laughter and similar devices can be a way of glossing over or implicitly explaining one's failure in everyday life to adhere to a scientific worldview, of which the rational choice model is an integral part. 
The points of view presented in section $\mathbf{3 C}$ constitute the most explicit reservations in the face of the prescriptive model of rational lifestyle choice found in our interviews. Here our informants, who through their enthusiastic work or through unselfish donations are committed to the idea that human genetics can provide relief and improve health, are explicit in their reservations against a lifestyle where prevention and 'tailoring' are the supreme measure of how one should lead one's life. Interestingly, ambivalence and reservations in the face of enthusiasm appear to be inherent features of their positions, rather than a temporary state of doubt to be resolved by an argument either in favour or in disfavour of human genetics.

Taking our lead from the statements "you have got to have a little freedom" and "you can worry yourself sick if you know too much", we believe that the ambivalent stance demonstrated by our informants can be explained in the light of van Hooft's theory of health. Human subjectivity is constituted not only by biology, but by the ways the organism relates to its environment, constructs meaning, partakes in social relationships and commits itself to certain ways of living, and genetic technologies designed to prevent or combat organic disease, can interfere with health on all these different levels. Thus, our informants' ambivalence can be understood as the logical consequence of reacting to genetics on two or more health levels at the same time: They are enthusiastic that genetic technologies can be useful to avert disease, but simultaneously suspicious that extensive predicting and 'tailoring' can have unintended and unfortunate effects on the creative processes involved in maintaining relatedness, purpose and meaning. Further, scientists and lay participants alike conducted their non-conclusive negotiations between contradictory opinions and sentiments in a quite identical manner, indicating perhaps that the basic requirements for assigning a meaningful and beneficial lifeworld role to medical technologies are shared by all subjects, and are not strongly influenced by technical expertise in the field in question. As reported in section $\mathbf{3 A}$ the prescriptive model of rational lifestyle choice emerged most clearly from the dialogue among the scientists, but their commitment to this model was balanced by equally strong statements of reservation, ambivalence and non-adherence indistinguishable from those of the lay participants.

As genetics is currently held to be among the most promising venues of research to further enhance the power of medical interventions against disease, pain and disability, any second thoughts about human genetics can be seen as spitting in the face of medicine's technical miracles. Further, medicine is thoroughly embedded in a scientific parlance of objectivity and disenchantment. Non-biological levels of health on the other hand, being intrinsically dependent on cultural and subjective interpretation, do hardly lend themselves to rigorous analysis and experimentation. Suggestions that genetics could be harmful to health - to meaning, agency, belonging, etc. - can therefore easily be dismissed as unscientific speculation, not suitable for rational validation or refutation, and therefore sheer nonsense. The very idea that regimes which effectively combat disease can be harmful to health is probably counter-intuitive to many. Our suggestion is that this results from the biological onedimensionality of Western medical and scientific epistemology: By neglect, this epistemology and the corresponding prescriptive beliefs currently in circulation do not distinguish, hence do not weigh benefits (or losses) on the biological dimension of disease against losses (or benefits) on non-biological levels of health. Implicitly, health is treated as a non-existent dimension in life; or rather the concept of health is 
reduced to imply merely the absence of disease, or the 'normal' functioning of organs. $^{19}$

Conventional risk communication has been shown to be at best an imperfect method for recruiting the general public to the regimes of preventive medicine. This has given rise to the understanding that individuals' and populations' health needs, values and aspirations must be explored as they provide the context within which the merits of medical technologies are to be established. Thus, it is an implication of our study that the non-biological dimensions of health should be taken into account when judging the proper role to be assigned to human genetic technologies. ${ }^{20}$

While our informants discussed the material costs of new technologies and the intellectual challenges involved in interpreting estimates of disease susceptibility, it seems to us that cultural resources in a broader sense may be equally important for people's ability to make sense of genetics. The decisions people make regarding their health are a product of their knowledge, imagination and sensitivity, vulnerable to anxiety, lack of insight and social support. Information and choices that may empower the privileged who have access to cultural resources to sort out their options, may be misinterpreted by others, causing suffering, loss of sense of good life, etc. Thus, one of the reasons why health is strongly related to class and social status ${ }^{21}$ appears to be that people need freedom, knowledge and social support to take good care of their health and to benefit from healthcare. We submit that this hinges on, among other things, people's ability to balance any unintended and unfavourable effects of medical technologies on the non-biological dimensions of health against their intended and favourable effect against biological disease.

In the face of van Hooft's model of health, it should be stressed that there is no way of knowing a priori the size or the sign of effects of genetic technologies on nonbiological levels of health. Indeed, returning to Tomas' and Thomas' theorem, it becomes clear that the public reception of genetic technologies in itself is medically relevant. And if the higher-level effects of new technologies are dependent on the cultural interpretation of the same technologies, the task of exploring and evaluating the higher-level effects of human genetics takes a creative turn: In addition to its analytical part, we need to mobilise the cultural and governance resources which will ensure the compatibility of genetic technologies with the full range of human subjectivity and health. This might produce some rather startling effects. Perhaps the private discourses of ambivalence and reflexivity may be regarded as a resource for the development of the required adaptation: their significance in this respect needs clarification in further research.

\section{Acknowledgements}

This study was supported by a grant from the Norwegian Research Council, grant no $147640 / 330$, and by a mobility scholarship from the Nordic Research Board, grant number 030444. The authors wish to thank the participants in the interviews for their generous assistance and the two anonymous reviewers for their valuable comments. 
${ }^{1}$ See for example: European Comission. 2003. Europeans and Biotechnology in 2002, Department of health. 2003. "Our inheritance, our future, realising the potential of genetics in the NHS", available at: http://www.doh.gov.uk/genetics/whitepaper.htm., and Wellcome Trust. 1998. Public perspectives on human cloning. London.

2 T. M. Bubela and T. A. Caulfield. Do the print media "hype" genetic research? A comparison of newspaper stories and peer-reviewed research papers. Cmaj. 2004;170(9):1399-1407.

${ }^{3}$ A. Petersen. Biofantasies: genetics and medicine in the print news media. Social Science \& Medicine. 2001;52(8):1255-1268.

${ }^{4} \mathrm{Cf}$. the project 'Public understanding of genetics': http://les1.man.ac.uk/sa/pug/index.htm. and B. Wynne. Creating public alientation: Expert cultures of risk and ethics on GMOs. Science as Culture. 2001;10(4):445-481.

${ }^{5}$ A classical account of reflexive modernity and the scientific construction/production of risk is found in: U. Beck. Risk society: towards a new modernity. London. Sage: 1992, while the following papers demonstrate how these ideas can be applied to the field of genetics and health: A. Kerr and S. Cunningham-Burley. On Ambivalence and Risk: Reflexive Modernity and the New Human Genetics. Sociology. 2000;34(2):283-304, and E. Beck-Gernsheim, Life as a planning project. In: S. Lash, B. Szerszynski and B. Wynne, eds. Risk, environment and modernity. London. Sage publications: 1996.

${ }^{6}$ Cf. L. Frewer. Risk perception, social trust, and public participation in strategic decision making: Implications for emerging technologies. Ambio. 1999;28(6):569-574, and J. G. Roederer.

Communicating with the public, politicians and the media. COSTED Occasional Paper. 1998;NO 1:128.

${ }^{7}$ A. Árnason and B. Simpson. Refractions through Culture: The New Genomics in Iceland. Ethnos. 2003;68(4):533-553. For balanced accounts of the controverses raised by Decode genetics see for example: G. Pálsson and K. E. Harðardóttir. For whom the cell tolls: Debates about biomedicine. Current anthropology. 2002;43(2):271-287, and J. F. Merz, G. E. McGee and P. Sankar. "Iceland Inc."? On the ethics of commercial population genomics. Soc Sci Med. 2004;58:1201-1209.

${ }^{8}$ European Comission. Special Eurobarometer 225: Social values, science \& technology. Available at: http://europa.eu.int/comm/public_opinion/archives/ebs/ebs_225_report_en.pdf.

${ }^{9}$ K. Kristjánsson, personal communication, May $12^{\text {th }}, 2004$

${ }^{10}$ Decoding the language of life - A case study of deCODE genetics in Iceland. Available at: http://www.uib.no/isf/people/stefan.htm.

${ }^{11}$ B. Wynne. May the sheep safely graze? A reflexive view of the expert-lay knowledge divide. In: Lash, Szerszynski and Wynne, op. cit. note 5.

12 B. Wynne. Creating public alientation: Expert cultures of risk and ethics on GMOs. Science as Culture. 2001;10(4):445-481.

${ }^{13}$ W. I. Thomas and D. S. Thomas. The Child in America: Behavior problems and programs. New York. Knopf: 1298.

${ }^{14}$ S. van Hooft. Health and subjectivity. Health. 1997;1(1):23-36, and S. van Hooft. Disease and subjectivity. In: J. M. Humber and R. F. Almeder, eds. What is disease? Totowa, NJ. Humana Press: 1997.

${ }^{15}$ Among the 13 scientists, there were 6 women and 7 men, 8 were Icelanders and 5 non-Icelanders, 4 were doctors and 9 biologists. The group of lay participants comprised 3 women and 1 man; the man stating that he had been recruited for Decode's research because he was affected by a certain disease; one of the women stated that she was related to someone suffering from a disease being researched; while the remaining two did not volunteer information about the reason for their recruitment for Decode's research. The inclusion of the lay participant group was approved by the Icelandic Research Ethics Committee, and their anonymity was secured by use of pseudonyms and exclusion from the transcript of any information from which their identity could be inferred.

${ }^{16}$ The general methodology applied is roughly equivalent to that described in R. Barbour and J. Kitzinger, eds. Developing focus group research. London. Sage Publications: 1999. For a closer description of the analytical strategy employed, see: R. B. Addison. A grounded hermeneutic editing approach. In: B. F. Crabtree and W. L. Miller, eds. Doing qualitative research. Thousand Oaks. Sage Publications: 1999, while the following paper by K. Malterud details some of the requirements for reflexivity on behalf of the investigator: Qualitative research: standards, challenges, and guidelines. Lancet. 2001;358(9280):483-488. 
${ }^{17}$ L. M. Silver. Remaking Eden: How genetic engineering and cloning will transform the American family. New York: Avon Books; 1998.

${ }^{18}$ While they were not asked to discuss genetic exceptionalism, our informants consistently maintained that genetic technologies being developed today will provide probabilistic prediction and prevention of disease in the same way as do conventional medical technologies. On this issue at least they agree with the current understanding that genetic information shares crucial features with other medical information. For a recent discussion of genetic exceptionalism in the context of common diseases, cf. the European commission's Expert group on genetic testing report Ethical, legal and social aspects of genetic testing: research, development and clinical applications. Brussels, 2004.

tic

${ }^{20}$ Ideally, the increasingly applied public health methods of Health Needs Assessment and Health Technology Assessment take into account cultural, social and ethical aspects of their subject matter, which we take to include acknowledgement that although immensely valuable to individuals and societies, the treatment and prevention of biological disease must also be evaluated in terms of its impact on other values, including the non-biological levels of health. The UK National Institute for Health and Clinical Excellence (NICE) provides an illustrating example of systematic and prospective assessment of the technical and public value of medical technologies, cf. especially http://www.publichealth.nice.org.uk/page.aspx?o=513203, accessed on December 14th 2005.

${ }^{21}$ Cf. R. G. Evans, T. R. Marmor and M. L. Barer, eds. Why are some people healthy and others not? New York. Aldine de Gruyter: 1994, and R. G. Wilkinson. Mind the gap: hierarchies, health and human evolution. New Haven. Yale University Press: 2001. 